\section{Greasy attractions}

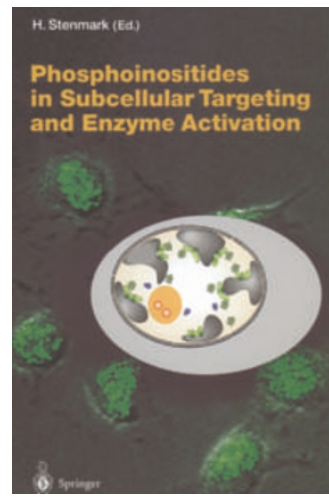

\section{Phosphoinositides in Subcellular Targeting and Enzyme Activation}

\section{Edited by Harald Stenmark}

Springer Verlag $\mathrm{GmbH} \bullet 2003$

$\$ 119 / £ 77$

The inositol ring is a simple structure, and within its simplicity lies its versatility: the ability to phosphorylate each of its six hydroxyls, individually and in combination, creating a range of molecules with distinct functions. Proteins have evolved to recognise specific combinations of these phosphates attached to the inositol ring and, remarkably, proteins can readily distinguish a phosphate attached to the $3^{\prime}, 4^{\prime}$ or $5^{\prime}$ hydroxyl group. Up to seven different phosphorylated inositol lipids are known to exist, and it now seems that cells make good use of this diversity. PH, FYVE, PX and ENTH domains, as well as short basic sequence motifs that reversibly bind phosphoinositides (PtdIns) with differing specificities and affinities, are found in many proteins in combination with other protein modules. The inositol lipid can act merely as a membrane-targeting device, or more commonly, result in a conformational change that may activate or inactivate a protein. The beauty of this regulatory system is in the rigorous control of production and destruction of specific lipids (how the balance of different phosphoinositides is maintained in different membrane compartments remains unknown). These and other recent advances in our understanding of phosphoinositides have now been summarized in a book edited by Harald Stenmark, Phosphoinositides in Subcellular Targeting and Enzyme Activation.

The book consists of seven chapters that focus on the various functions of PtdIns. PtdIns-mediated protein recruitment regulates many cellular processes including chemotaxis, phagocytosis, endocytosis and organization of the actin cytoskeleton. Thus, these are the major cellular functions that this book focuses on. The cells of the immune system are often cited as examples of the multiple uses of these phosphorylated lipids in subcellular targeting and regulation of enzyme activity. However, this should not deter the cell biologist interested in inositide biology, as these principles are applicable to all cells.

Shamshad Cockcroft, Lipid Signalling Group, Department of Physiology, University College London, London WC1E 6JJ.

e-mail:ucgbsxc@ucl.ac.uk
Phagocytosis is a process in which cells of the immune system remove invading micro-organisms. Sergio Grinstein and colleagues takes us through the process of phagocytosis and introduce the reader to PtdIns $(4,5) \mathrm{P}_{2}$; an accumulation of this lipid at the phagosomal cup allows the recruitment of the actin cytoskeleton. Subsequently, PtdIns $(4,5) \mathrm{P}_{2}$ disappears as some of it is converted into PtdIns $(3,4,5) \mathrm{P}_{3}$ and some is degraded by phospholipase C. This accomplishes two things: the removal of $\operatorname{PtdIns}(4,5) \mathrm{P}_{2}$, leading to the dismantling of the actin meshwork, and the production of PtdIns $(3,4,5) \mathrm{P}_{3}$, which then attracts a new set of proteins to take the nascent phagosome to the next stage in its journey. This chapter provides a comprehensive guide to the dynamic nature of the process. The phagosome is equivalent to the endosome, and Harald Stenmark provides an in-depth discussion of the targeting of specific proteins using the FYVE and PX domains to endosomal compartments by binding phospholipids on these organelles. Stenmark also describes the structural basis for lipid-protein interactions, and provides the rational basis for these interactions.

The actin cytoskeleton is fundamental to many cellular processes, including cell motility, phagocytosis, morphogenesis, endo- and exocytosis, cytokinesis and neurite extension. There is a vast array of actin-associated proteins that are regulated mainly by $\operatorname{PtdIns}(4,5) \mathrm{P}_{2}$ and a few by the 3-phosphoinositides. Pekka Lappallainen succinctly summarises our understanding of the specific actin cytoskeletal proteins that are regulated by PtdIns. We are given a tour of proteins that cap and sever actin filaments, actin-monomer-binding proteins, proteins that promote actin filament nucleation and proteins that crosslink actin.

The $\mathrm{PH}$ domain has pride of place, as it was the first protein domain that was recognised to bind PtdIns $(4,5) \mathrm{P}_{2}$. Peter Cullen and colleagues update our knowledge of different $\mathrm{PH}$ domains, and how they interact with PtdIns. In the human genome the $\mathrm{PH}$ domain is the eleventh most common domain, and there are $252 \mathrm{PH}$-domain proteins in total. It is becoming evident that the majority of $\mathrm{PH}$ domains do not bind PtdIns with high affinity; this may be the exception rather than the rule, and low-affinity interactions may be more common. But lowaffinity interactions may operate in the presence of a second low affinity interaction with another ligand, which could be protein or lipid.

The final chapter takes us into the nucleus - where the inositol lipids are not found in a membrane, but are actually free within the nucleus - and provides useful background information for nonexperts. What inositol lipids and the water-soluble inositol phosphates are doing in the nucleus remains a mystery, and this chapter discusses many possible ideas.

Although this book places a strong emphasis on how PtdIns regulate proteins, it fails to provide any details on the machinery that regulates the production and destruction of these lipids. However, this book summarises recent advances in the burgeoning field of inositol lipids and provides a snapshot of where the field stands in 2003. The field is moving rapidly but this volume will be a useful addition for many researchers who have only recently discovered that their favourite protein is regulated by PtdIns. 